\title{
Alignment of quasar polarizations with large-scale structures ${ }^{\star} \star \star \star$
}

\author{
D. Hutsemékers ${ }^{1}$, L. Braibant ${ }^{1}$, V. Pelgrims ${ }^{1}$, and D. Sluse ${ }^{2}$ \\ ${ }^{1}$ Institut d'Astrophysique et de Géophysique, Université de Liège, Allée du 6 Août 17, B5c, 4000 Liège, Belgium \\ e-mail: hutsemekers@astro.ulg.ac.be \\ 2 Argelander-Institut für Astronomie, Auf dem Hügel 71, 53121 Bonn, Germany
}

Received 18 July 2014 / Accepted 7 September 2014

ABSTRACT

\begin{abstract}
We have measured the optical linear polarization of quasars belonging to Gpc scale quasar groups at redshift $z \sim 1.3$. Out of 93 quasars observed, 19 are significantly polarized. We found that quasar polarization vectors are either parallel or perpendicular to the directions of the large-scale structures to which they belong. Statistical tests indicate that the probability that this effect can be attributed to randomly oriented polarization vectors is on the order of $1 \%$. We also found that quasars with polarization perpendicular to the host structure preferentially have large emission line widths while objects with polarization parallel to the host structure preferentially have small emission line widths. Considering that quasar polarization is usually either parallel or perpendicular to the accretion disk axis depending on the inclination with respect to the line of sight, and that broader emission lines originate from quasars seen at higher inclinations, we conclude that quasar spin axes are likely parallel to their host large-scale structures.
\end{abstract}

Key words. large-scale structure of Universe - quasars: general

\section{Introduction}

Hutsemékers et al. (1998, 2001, 2005, hereafter Papers I-III) have reported alignments of quasar optical linear polarizations extending over Gpc scale regions of the sky at redshift $z \sim 1$ (see also Jain et al. 2004; Shurtleff 2013; Pelgrims \& Cudell 2014). Possible effects modifying the polarization of light along the line of sight, in particular mixing with axion-like particles, have been investigated in detail (e.g., Das et al. 2005; Agarwal et al. 2012). However, because of the absence of comparable circular polarization, these mechanisms have been essentially ruled out (Hutsemékers et al. 2010; Payez et al. 2011).

Since quasar polarization is often related to the object's geometry, another interpretation would be that quasars themselves are aligned, presumably with the structure to which they belong. To test this hypothesis, we have measured the polarization of quasars belonging to the large quasar group (LQG) constituted of the groups U1.27 (aka Huge-LQG) and U1.28 (aka CCLQG) described in Clowes et al. (2013). These quasar structures extend over Gpc scales at redshift $z \sim 1.3$, possibly beyond the homogeneity scale of the concordance cosmology ( $\sim 370 \mathrm{Mpc}$, Yadav et al. 2010; but see Nadathur 2013; Einasto et al. 2014).

\section{Observations and polarization measurements}

Observations were carried out at the European Southern Observatory, Paranal, on March 22-26, 2014, using the Very Large Telescope equipped with the FORS2 intrument in the standard imaging polarimetry mode $\mathrm{IPOL}^{1}$. Linear polarimetry is performed by inserting in the parallel beam a Wollaston prism which splits the incoming light rays into two orthogonally

* Based on observations made with ESO Telescopes at the La Silla Paranal Observatory under program ID 092.A-0221.

$\star \star$ Table 1 is available in electronic form at http://www . aanda.org

1 FORS User Manual, VLT-MAN-ESO-13100-1543, Issue 92.0. polarized beams separated by $22^{\prime \prime}$. Image overlapping is avoided by inserting a special mask in the focal plane. To measure the normalized Stokes parameters $q$ and $u, 4$ frames are obtained with the half-wave plate rotated at 4 position angles, $0^{\circ}, 22.5^{\circ}$, $45^{\circ}$, and $67.5^{\circ}$. This procedure allows us to remove most of the instrumental polarization. The linear polarization degree $p$ and position angle $\theta$ are derived using $p=\left(q^{2}+u^{2}\right)^{1 / 2}$ and $\theta=1 / 2 \arctan (u / q)$ so that $q=p \cos 2 \theta$ and $u=p \sin 2 \theta$. Since orthogonally polarized images of the object are simultaneously recorded, the measured polarization does not depend on variable transparency or seeing.

All observations were obtained using the FORS2 V high filter $\left(\lambda_{0}=555 \mathrm{~nm}, F W H M=123 \mathrm{~nm}\right)$. Data reduction and measurements were performed as detailed in Sluse et al. (2005). The instrumental polarization was checked using the unpolarized stars WD0752-676 and WD1615-154 (Fossati et al. 2007) and found to be $p=0.05 \pm 0.06 \%$, which is consistent with zero $^{2}$. We did not use field stars to estimate the instrumental polarization because of spurious off-axis polarization in FORS1/2 (Patat \& Romaniello 2006). To fix the zero-point of the polarization position angle, polarized standard stars have been observed: NGC 2024-1, Ve 6-23, CD-28 13479, HD 316232, BD-14 922 (Fossati et al. 2007). The offset - to subtract from the raw polarization angle - was determined to be $2.5^{\circ} \pm 0.5^{\circ}$ in the V_high filter.

The linear polarization of all 73 quasars of the Huge-LQG and of 20 out of the 34 quasars of the CCLQG has been obtained, i.e., for a total of 93 quasars. Table 1 summarizes the measurements. The error on the polarization degree is between $0.06 \%$ and $0.23 \%$, with a mean value of $0.12 \%$. The distribution of the debiased polarization degree is illustrated in Fig. 1. It shows a peak near the null value, in agreement with other polarization measurements of radio-quiet non-BAL

\footnotetext{
2 We also observed HD 64299 which turned out to be polarized with $p=0.17 \pm 0.04 \%$, in agreement with Masiero et al. (2007).
} 


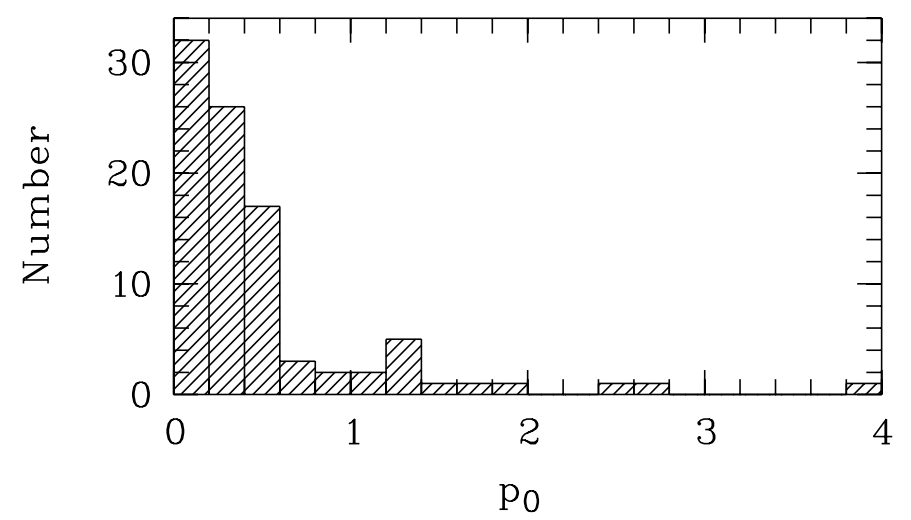

Fig. 1. Distribution of the debiased polarization degree $p_{0}$ (in $\%$ ) measured for the sample of 93 quasars.

quasars (Berriman et al. 1990; Hutsemékers et al. 1998b). All objects are at galactic latitudes higher than $50^{\circ}$ which minimizes contamination by interstellar polarization. In this region of the sky, the interstellar polarization is around $p_{\text {is }} \simeq 0.1 \%$ with a peak near $50^{\circ}$ (Fig. 2). As in Papers I-III, we consider that polarization is essentially intrinsic to the quasar when $p \geq 0.6 \%$ (Berriman et al. 1990; Hutsemékers et al. 1998b; Sluse et al. 2005). Out of 93 quasars, 19 have $p \geq 0.6 \%$. Their properties are given in Table 2 . For these 19 polarized quasars, $\sigma_{\theta} \leq 10^{\circ}$ with an average value around $3^{\circ}$.

\section{Analysis of polarization alignments}

In Fig. 3 we show a map of the quasar polarization vectors over the LQG structure. The map does not show any evidence of coherent orientations or alignments. The distribution of the polarization angles is flat, compatible with random orientations and with no contamination by interstellar polarization.

In order to compare the quasar polarization angles to the direction of the local structures, we consider four structures for which we determine a mean orientation, as illustrated in Fig. 4. Group 4 is the CCLQG defined in Clowes et al. (2012). The Huge-LQG is divided in groups denoted 1, 2 and 3. Group 3 corresponds to the branch set of 17 quasars identified by Clowes et al. (2013). The large vertical part of the Huge-LQG is then separated into groups 1 and 2. The mean projected direction of the structures is determined by an orthogonal regression in right ascension, declination (Isobe et al. 1990). For groups 1-4, we measure the position angles $\mathrm{PA}=157^{\circ}, 164^{\circ}, 81^{\circ}$, and $109^{\circ}$, respectively. We estimate the acute angle between the quasar polarization vectors and the PA of the structures to which they belong using $\Delta \theta=\min \left(|\mathrm{PA}-\theta|, 180^{\circ}-|\mathrm{PA}-\theta|\right)$.

The distribution of $\Delta \theta$ is illustrated in Fig. 5. It shows a bimodal distribution, with both alignments $\left(\Delta \theta \simeq 0^{\circ}\right)$ and antialignments $\left(\Delta \theta \simeq 90^{\circ}\right)$ in each quasar group (except group 3). The cumulative binomial probability of having nine or more quasars in the first and the last bins is $P_{\text {bin }}=1.4 \%$. The Kuiper test (Arsham 1988; Fisher 1993) gives a probability $P_{K}=1.6 \%$ that the observed distribution is drawn from an uniform distribution. These results are robust if we consider the 28 quasars with $p \geq 0.5 \%$ (in this case $P_{\text {bin }}=1.2 \%$ and $P_{K}=1.0 \%$ ).

A bimodal distribution of $\Delta \theta$ is exactly what we expect if the quasar morphological axes are related to the orientation of the host large-scale structures. Indeed, the polarization of type 1 AGN is usually either parallel or perpendicular to the AGN accretion disk axis depending on the inclination with respect to the
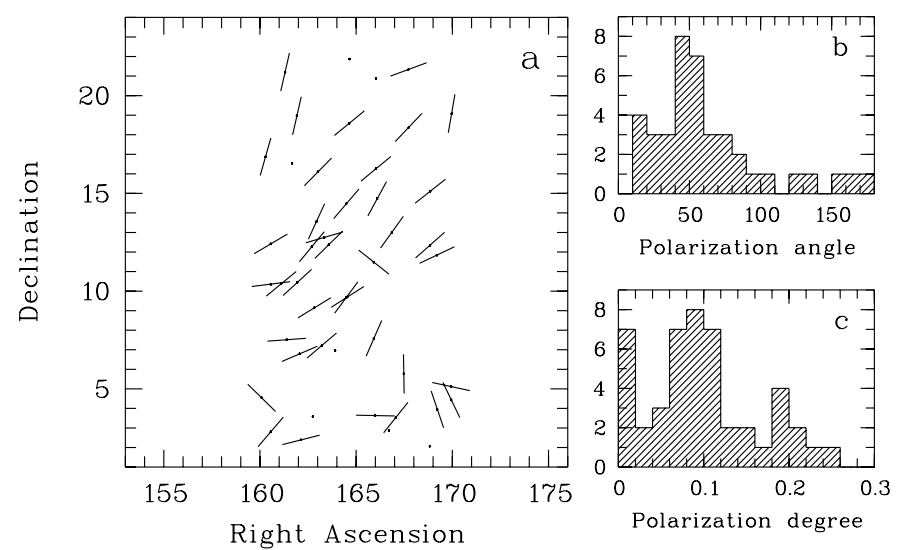

Fig. 2. Interstellar polarization in the region of the sky corresponding to the quasar large-scale structure under study (data from Berdyugin et al. 2014). a) Map of polarization vectors; right ascensions and declinations are in degree; the length of the polarization vectors is arbitrary. b) Distribution of polarization angles (in degree). c) Distribution of polarization degrees (in \%).

line of sight (e.g., Smith et al. 2004). We may assume that higher luminosity AGN (quasars) behave similarly. In Fig. 6, the quasar polarization angles modified according to $\widetilde{\theta}=\bmod \left(\theta, 90^{\circ}\right)+90^{\circ}$ are plotted over the LQG structure, unveiling a remarkable correlation. We stress that such a behavior cannot be due to contamination by interstellar polarization which would align all polarizations similarly.

To quantify the significance of this correlation independently of the shape of the host structure, we use the Andrews and Wasserman $Z_{c}$ statistical test (Bietenholz 1986, Paper I). This test is best suited to small samples since it does not involve angle dispersion. The idea of the Andrews \& Wasserman test is to compute for each object $i$, the mean direction $\bar{\theta}_{i}$ of its $n_{v}$ nearest neighbors, and to compare this local average to the polarization angle of the object $i, \theta_{i}$. If angles are correlated to positions, one expects, on the average, $\theta_{i}$ to be closer to $\bar{\theta}_{j=i}$ than to $\bar{\theta}_{j \neq i}$. As a measure of the closeness of $\theta_{i}$ and $\bar{\theta}_{j}$, one uses $D_{i, j}=\boldsymbol{y}_{i} \cdot \overline{\boldsymbol{Y}}_{j}$, where $\boldsymbol{y}_{i}$ is the normalized polarization vector of object $i$ and $\overline{\boldsymbol{Y}}_{j}$ the normalized resultant polarization vector of the $n_{v}$ neighbors of object $j$, excluding $j$. Then $Z_{i}$ is computed by ranking $D_{i, j=i}$ among the $D_{i, j=1, n}$ and the final statistics $Z_{\mathrm{c}}$ is obtained by averaging the $Z_{i}$ over the sample of $n$ objects. $Z_{\mathrm{c}}$ is expected to be significantly larger than zero when the polarization angles are not randomly distributed over positions. To make the test independent of the coordinate system, polarization vectors can be parallel transported before computing the resultant polarization vectors (Jain et al. 2004). Here, the polarization vectors are computed using $\boldsymbol{y}=(\cos \Theta$, $\sin \Theta)$ with $\Theta=4 \bmod \left(\theta, 90^{\circ}\right)$ instead of $\Theta=2 \theta=2 \bmod \left(\theta, 180^{\circ}\right)$ to test for either alignments or anti-alignments (i.e., dealing with 4-axial data instead of 2-axial data, Fisher 1993). To estimate the statistical significance, $10^{5}$ samples of 19 angles were created through Monte-Carlo simulations either by shuffling the measured angles over positions, or by randomly generating them in the $\left[0^{\circ}, 180^{\circ}\right]$ range (Press et al. 1992). The significance level (S.L.) of the test is finally computed as the percentage of simulated configurations for which $Z_{\mathrm{c}} \geq Z_{\mathrm{c}}^{\star}$ where $Z_{\mathrm{c}}^{\star}$ is the measured sample statistics. Since all quasars are in a limited redshift range, we only consider their angular positions on the sphere.

The significance level of the $Z_{\mathrm{c}}$ test is illustrated in Fig. 7. It shows that the probability that the polarization angles are randomly distributed over positions is smaller than $1 \%$. The effect 
Table 2. Sample of 19 quasars with $p \geq 0.6 \%$.

\begin{tabular}{lcrrrrrrr}
\hline \hline Object & LQG & $\begin{array}{r}p \\
(\%)\end{array}$ & $\begin{array}{r}\sigma_{p} \\
(\%)\end{array}$ & $\begin{array}{r}\theta \\
\left({ }^{\circ}\right)\end{array}$ & $\begin{array}{r}\sigma_{\theta} \\
\left({ }^{\circ}\right)\end{array}$ & $\begin{array}{r}F W H M \\
\mathrm{~km} \mathrm{~s}^{-1}\end{array}$ & $\begin{array}{r}\sigma_{\mathrm{FWHM}} \\
\mathrm{km} \mathrm{s}^{-1}\end{array}$ \\
\hline SDSSJ105421.90+212131.2 & 1.2573 & 1 & 1.04 & 0.08 & 92.6 & 2.2 & 5094 & 214 \\
SDSSJ105446.73+195710.5 & 1.2195 & 1 & 1.89 & 0.23 & 75.2 & 3.5 & 3256 & 363 \\
SDSSJ105611.27+170827.5 & 1.3316 & 1 & 1.29 & 0.08 & 44.8 & 1.8 & 6088 & 158 \\
SDSSJ110016.88+193624.7 & 1.2399 & 1 & 1.14 & 0.23 & 160.4 & 5.9 & 3909 & 348 \\
SDSSJ104445.03+151901.6 & 1.2336 & 2 & 1.25 & 0.11 & 167.5 & 2.5 & 3254 & 196 \\
SDSSJ104616.31+164512.6 & 1.2815 & 2 & 1.25 & 0.11 & 86.9 & 2.5 & 2635 & 222 \\
SDSSJ104859.74+125322.3 & 1.3597 & 2 & 0.72 & 0.13 & 45.6 & 5.3 & 3746 & 397 \\
SDSSJ104941.67+151824.6 & 1.3390 & 2 & 1.31 & 0.13 & 146.4 & 2.9 & 4034 & 633 \\
SDSSJ105245.80+134057.4 & 1.3544 & 2 & 1.32 & 0.11 & 30.2 & 2.4 & 5885 & 174 \\
SDSSJ105442.71+104320.6 & 1.3348 & 2 & 0.73 & 0.11 & 172.8 & 4.4 & 4108 & 269 \\
SDSSJ105525.68+113703.0 & 1.2893 & 2 & 2.55 & 0.10 & 49.1 & 1.1 & 4443 & 399 \\
SDSSJ11009.58+075206.8 & 1.2123 & 3 & 1.81 & 0.17 & 34.2 & 2.7 & 5032 & 626 \\
SDSSJ111802.11+103302.4 & 1.2151 & 3 & 3.97 & 0.10 & 142.4 & 0.7 & 6900 & 1256 \\
SDSSJ104116.79+035511.4 & 1.2444 & 4 & 1.55 & 0.11 & 99.7 & 2.0 & 2195 & 296 \\
SDSSJ104225.63+035539.1 & 1.2293 & 4 & 0.69 & 0.08 & 23.2 & 3.3 & 5182 & 380 \\
SDSSJ105010.05+043249.1 & 1.2158 & 4 & 2.67 & 0.08 & 101.5 & 0.9 & 2703 & 190 \\
SDSSJ105512.23+061243.9 & 1.3018 & 4 & 0.98 & 0.12 & 115.9 & 3.5 & 3381 & 299 \\
SDSSJ105833.86+055440.2 & 1.3222 & 4 & 0.62 & 0.21 & 37.8 & 10.3 & 5167 & 410 \\
SDSSJ110108.00+043849.6 & 1.2516 & 4 & 0.84 & 0.10 & 25.7 & 3.4 & 4823 & 269 \\
\hline
\end{tabular}

Notes. Column 1 gives the quasar SDSS name; Col. 2 the redshift $z$; Col. 3 the quasar group (Fig. 4); Cols 4 and 5 the polarization degree $p$ and its error $\sigma_{p}$; Cols 6 and 7 the polarization position angle $\theta$ and its error $\sigma_{\theta}$; Cols. 8 and 9 the MgII emission line FWHM and its error from Shen et al. (2011).
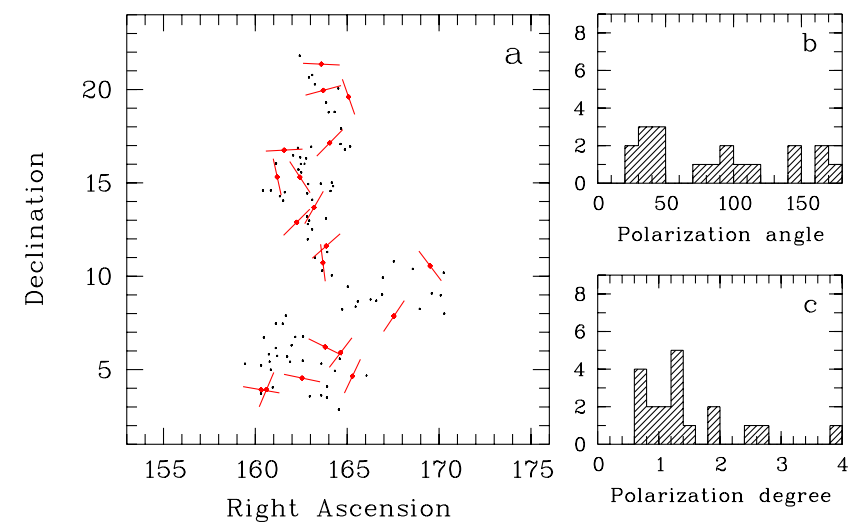

Fig. 3. Polarization of the 19 quasars with $p \geq 0.6 \%$. a) Map of polarization vectors over the large-scale structure; right ascensions and declinations are in degree; the length of the polarization vectors is arbitrary. b) Distribution of polarization angles (in degree). c) Distribution of polarization degrees (in \%).

is stronger $($ S.L. $<0.1 \%)$ when the mean orientation is computed with 10 nearest neighbors, i.e., roughly half of the sample. This number corresponds to a mean comoving distance of $\sim 550 \mathrm{Mpc}$, in agreement with the trend seen in Fig. 6. Parallel transport has little effect since all quasars lie close to each other and to the equator. We emphasize that a deviation from uniformity is only detected when using $4 \bmod \left(\theta, 90^{\circ}\right)$ in the $Z_{\mathrm{c}}$ test and not when using $2 \bmod \left(\theta, 180^{\circ}\right)$, which means that purely parallel or perpendicular alignments are not seen (Fig. 3). If we consider the 28 quasars with $p \geq 0.5 \%$, a similar curve is obtained with the minimum shifted to $n_{v}=20$, which corresponds to a mean comoving distance of $\sim 650 \mathrm{Mpc}$.

Since the width of low-ionization emission lines $(\mathrm{H} \beta, \mathrm{MgII})$ observed in quasar spectra correlates with the object's inclination with respect to the line of sight (Wills \& Brown 1986; Brotherton 1996; Jarvis \& McLure 2006; Decarli et al. 2008), we plot in Fig. 8 the angle $\Delta \theta$ as a function of the quasar

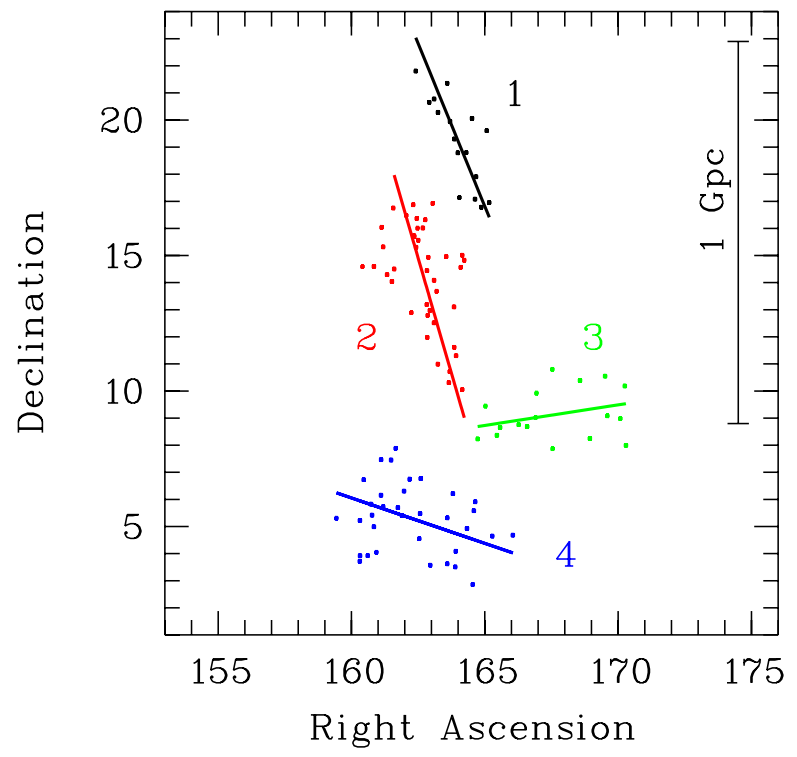

Fig. 4. Quasar groups and their orientations on the sky. Right ascensions and declinations are in degree. The superimposed lines illustrate the orientations of the four groups labeled 1,2,3,4. The comoving distance scale at redshift $z=1.3$ is indicated assuming a flat Universe with $H_{0}=$ $70 \mathrm{~km} \mathrm{~s}^{-1} \mathrm{Mpc}^{-1}$ and $\Omega_{\mathrm{m}}=0.27$.

MgII emission line width (FWHM from Shen et al. 2011). We see that most objects with polarization perpendicular to the host structure $\left(\Delta \theta>45^{\circ}\right)$ have large emission line widths while all objects with polarization parallel to the host structure $\left(\Delta \theta<45^{\circ}\right)$ have small emission line widths. A two-sample KolmogorovSmirnov test indicates that there is a probability of only $1.4 \%$ that quasars with either perpendicular or parallel polarizations have emission line widths drawn from the same parent population. Quasars seen at higher inclinations ${ }^{3}$ generally show

Face-on: $i=0^{\circ}$. Edge-on: $i=90^{\circ}$. 


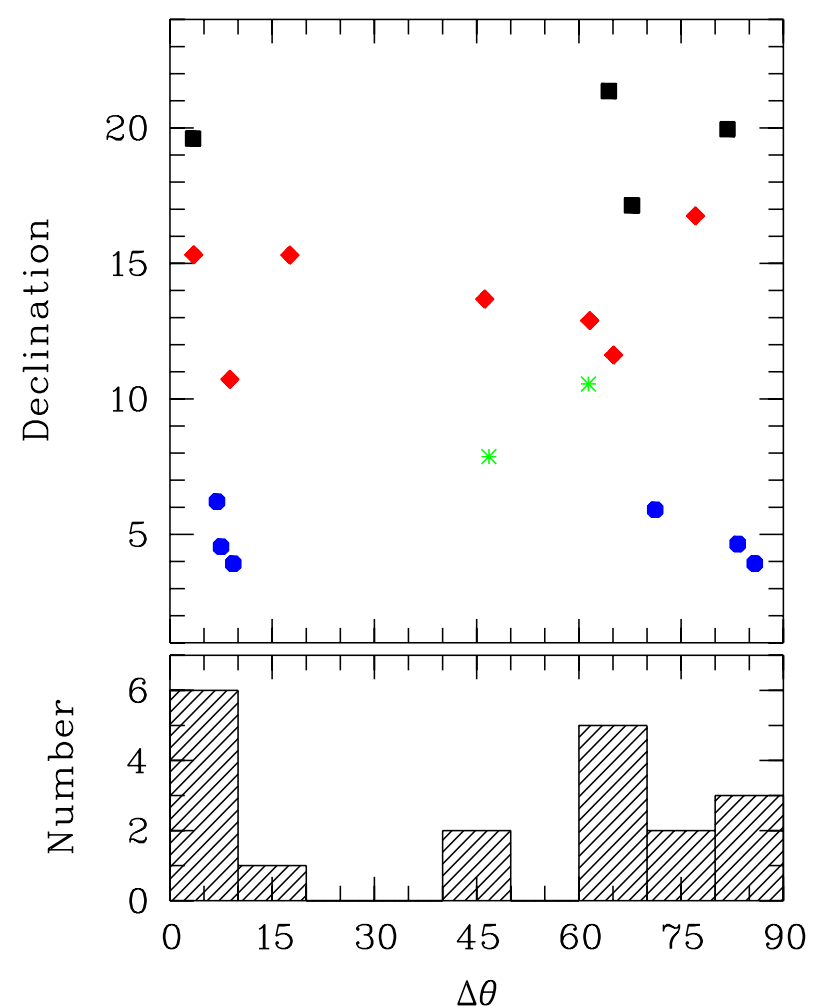

Fig. 5. Bottom: distribution of the acute angle $\Delta \theta$ (in degree) between quasar polarizations and the orientation of their host large-scale structure. Top: $\Delta \theta$ is plotted against the object's declination (in degree) to illustrate the behavior of the different quasar groups (1: squares; 2: lozenges; 3: asterisks; 4: hexagons; colors as in Fig. 4).

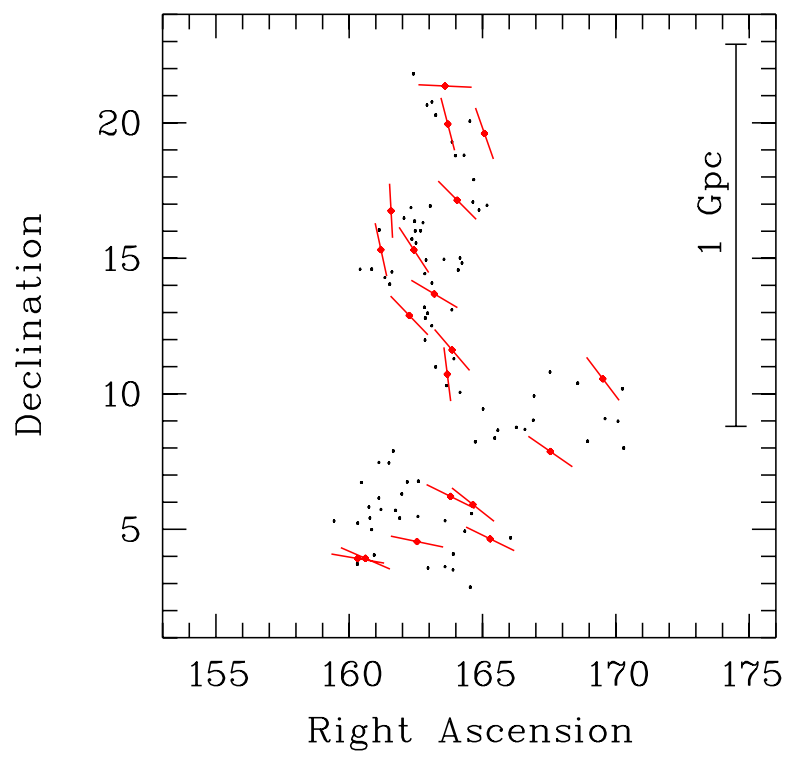

Fig. 6. Polarization vectors of the 19 quasars with $p \geq 0.6 \%$ are superimposed on the large-scale structure after rotation of the polarization angles according to $\tilde{\theta}=\bmod \left(\theta, 90^{\circ}\right)+90^{\circ}$. A clear correlation is seen but we nevertheless caution against exaggerated visual impression since polarization angles are now in the range $\left[90-180^{\circ}\right]$. Right ascensions and declinations are in degree. The comoving distance scale is indicated as in Fig. 4.

broader low-ionization emission lines, in agreement with line formation in a rotating disk (Wills \& Brown 1986; Jarvis \& McLure 2006; Decarli et al. 2008). The relation seen in Fig. 8

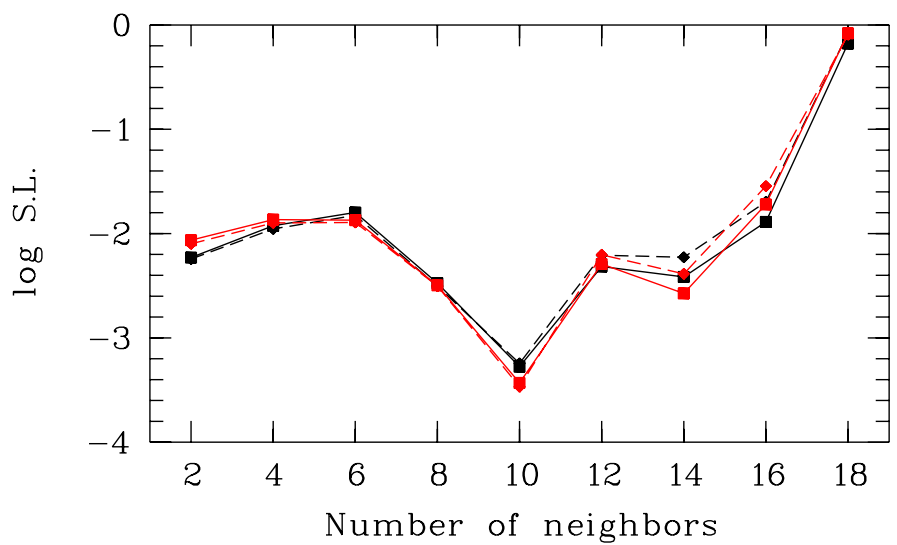

Fig. 7. Logarithm of the significance level (S.L.) of the $Z_{\mathrm{c}}$ test applied to the sample of 19 polarized quasars, as a function of the number of nearest neighbors. The solid line refers to simulations obtained by shuffling angles over positions while the dashed line refers to simulations obtained by randomly generating angles. The statistics are computed with (in red) and without (in black) parallel transport of the polarization vectors.

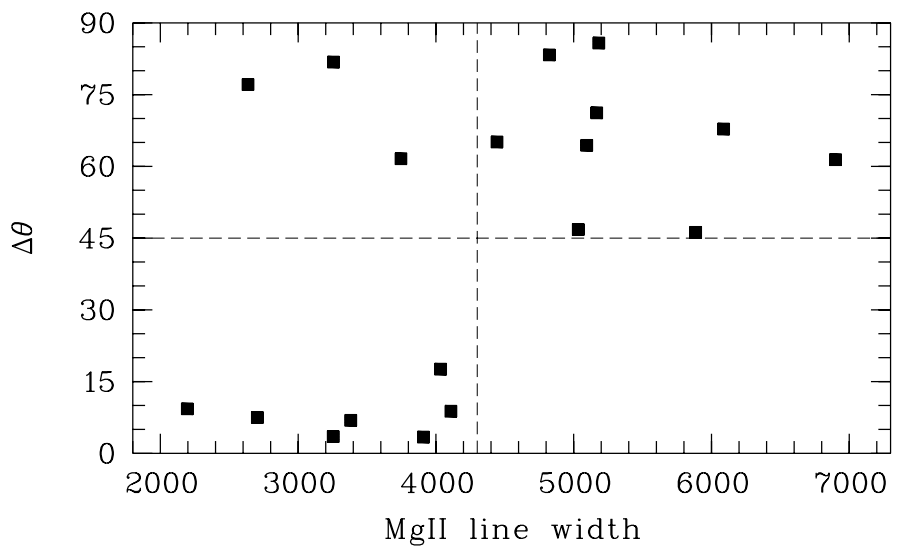

Fig. 8. Angle $\Delta \theta$ (in degree) between quasar polarizations and the orientation of their host large-scale structures as a function of the MgII emission line width (FWHM in $\mathrm{km} \mathrm{s}^{-1}$ ).

thus supports our hypothesis that the polarization of quasars is either parallel or perpendicular to the host structure depending on their inclination. When rotating by $90^{\circ}$ the polarization angles of objects with MgII emission line widths larger than $4300 \mathrm{~km} \mathrm{~s}^{-1}$, a stronger alignment is seen (Fig. 9). The Kuiper test gives a probability $P_{K}=0.5 \%$ that the observed distribution is drawn from an uniform distribution, but this value should be seen with caution since the cut at $4300 \mathrm{~km} \mathrm{~s}^{-1}$ is arbitrary. On the other hand, it should be emphasized that the emission line width does not only depend on inclination but also on the mass of the central black hole if the rotating disk is virialized. Quasars with lower black hole mass will have narrower emission lines whatever their inclination so that some of them may still appear anti-aligned in Fig. 9.

Since objects seen at higher inclinations preferentially show polarization perpendicular to their axes (Smith et al. 2004), we finally infer that quasar spin axes should be predominantly parallel to the orientation of the structures to which they belong. 
D. Hutsemékers et al.: Alignment of quasar polarizations with large-scale structures

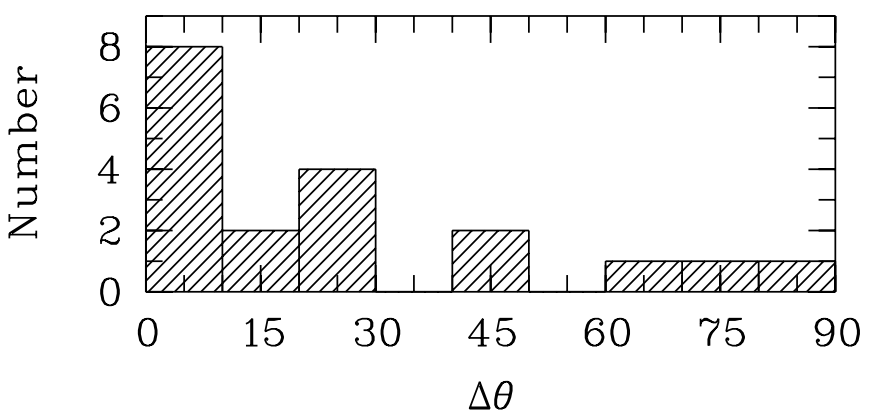

Fig. 9. Distribution of the acute angle $\Delta \theta$ (in degree) between quasar polarizations and the orientation of their host large-scale structure after rotating by $90^{\circ}$ the polarization angles of objects with MgII emission line widths larger than $4300 \mathrm{~km} \mathrm{~s}^{-1}$.

\section{Conclusions}

We have measured the polarization of 93 quasars belonging to large-scale quasar groups. 19 quasars out of 93 are significantly polarized with $p \geq 0.6 \%$.

We found that quasar polarization vectors are either parallel or perpendicular to the large-scale structures to which they belong, and correlated to the polarization vectors of their neighbors. The probability that these results can be attributed to a random distribution of polarization angles is on the order of $1 \%$. Such a behavior cannot be due to contamination by interstellar polarization. Our results are robust if we consider $p \geq 0.5 \%$ instead of $p \geq 0.6 \%$, or if we subtract a systematic $p_{\text {is }}=0.1 \%$ at $\theta_{\text {is }}=50^{\circ}$ to simulate the correction of a possible contamination by interstellar polarization (Fig. 2).

Assuming that quasar polarization is either parallel or perpendicular to the accretion disk axis as a function of inclination, as observed in lower luminosity AGN, and considering that broader emission lines originate from quasars seen at higher inclinations, we inferred that quasar spin axes are likely parallel to their host large-scale structures.

Galaxy spin axes are known to align with large-scale structures such as cosmic filaments (e.g., Tempel \& Libeskind 2013; Zhang et al. 2013, and references therein). Till now, such alignments are detected up to redshift $z \sim 0.6$ at scales $\lesssim 100 \mathrm{Mpc}(\mathrm{Li}$ et al. 2013). Detailed interpretations remain complex because the link between galaxy and halo spin axes is not straightforward, and because the strength and orientation of the alignments depend on several factors, in particular the mass of the halo and the cosmic history (e.g., Hahn et al. 2010; Trowland et al. 2013; Dubois et al. 2014). We have found that quasar accretion disk axes are likely parallel to the large-scale structures to which they belong over Gpc scales at redshift $z \sim 1.3$, i.e., one order of magnitude bigger than currently known galaxy alignments. Although the scales involved are much larger, we may assume that similar mechanisms can explain alignments of quasar and galaxy axes with their host large-scale structure, keeping in mind that polarization-related quasar regions (accretion disk, jet, scattering region) are not necessarily well aligned with the stellar component of the host galaxy (Borguet et al. 2008; Hopkins et al. 2012), and that quasars, more prone to strong feedback mechanisms, can have a different cosmic history (Dubois et al. 2014).

Since coherent orientations of quasar polarization vectors, and then quasar axes, are found on scales larger than $500 \mathrm{Mpc}$, our results might also provide an explanation to the very largescale polarization alignments reported in Papers I-III. In this case those alignments would be intrinsic and not due to a modification of the polarization along the line of sight. The existence of correlations in quasar axes over such extreme scales would constitute a serious anomaly for the cosmological principle.

Acknowledgements. We thank the referee, E. Tempel, for constructive remarks which helped to improve the paper. We thank B. Borguet, R. Cabanac, J.R. Cudell, H. Lamy and A. Payez for discussions and V. Ivanov for his efficient help during the observations. D.H. and L.B. are respectively Senior Research Associate and Research Assistant at the F.R.S.-FNRS. D.S. acknowledges support from the Deutsche Forschungsgemeinschaft, reference SL172/1-1. This work was supported by the Fonds de la Recherche Scientifique - FNRS under grant 4.4501.05. This research has made use of the NASA/IPAC Extragalactic Database (NED) which is operated by the Jet Propulsion Laboratory, California Institute of Technology, under contract with the National Aeronautics and Space Administration.

\section{References}

Agarwal, N., Aluri, P. K., Jain, P., Khanna, U., \& Tiwari, P. 2012, EPJC, 72, 1928

Arsham H. 1988, J. Appl. Stat., 15, 131

Berdyugin, A., Piirola, V., \& Teerikorpi, P. 2014, A\&A, 561, A24

Berriman, G., Schmidt, G. D., West, S .C., \& Stockman, H. S. 1990, ApJS, 74, 869

Bietenholz, M. F. 1986, AJ, 91, 1249

Borguet, B., Hutsemékers, D., Letawe, G., Letawe, Y., \& Magain, P. 2008, A\&A, 478, 321

Brotherton, M. S. 1996, ApJS, 102, 1

Clowes, R. G., Campusano, L. E., Graham, M. J., \& Söchting, I. K. 2012, MNRAS, 419, 556

Clowes, R. G., Harris, K. A., Raghunathan, S., et al. 2013, MNRAS, 429, 2910

Das, S., Jain, P., Ralston, J. P., \& Saha, R. 2005, J. Cosmology Astropart. Phys., 06, 002

Decarli, R., Labita, M., Treves, A., \& Falomo, R. 2008, MNRAS, 387, 1237

Dubois, Y., Pichon, C., Welker, C., et al. 2014, MNRAS, 444, 1453

Einasto, M., Tago, E., Lietzen, H., et al., 2014, A\&A, 568, A46

Fisher, N. I. 1993, Statistical Analysis of Circular Data (Cambridge: Cambridge University Press)

Fossati, L., Bagnulo, S., Mason, E., \& Landi Degl-Innocenti, E. 2007, ASP Conf. Ser., 364, 503

Hahn, O., Teyssier, R., \& Marcella Carollo, C. 2010, MNRAS, 405, 274

Hopkins, P. F., Hernquist, L., \& Hayward, C. C. 2012, MNRAS, 425, 1121

Hutsemékers, D. 1998, A\&A, 332, 410 (Paper I)

Hutsemékers, D., \& Lamy, H. 2001, A\&A, 367, 381 (Paper II)

Hutsemékers, D., Lamy, H., \& Remy, M. 1998, A\&A, 340, 371

Hutsemékers, D., Lamy, H., Cabanac, R., \& Sluse, D. 2005, A\&A, 441, 915 (Paper III)

Hutsemékers, D., Borguet, B., Sluse, D., Cabanac, R., \& Lamy, H. 2010, A\&A, 520, L7

Isobe, T., Feigelson, E., Akritas, M. G., \& Babu, G. J. 1990, ApJ, 364, 104

Jain, P., Narain, G., \& Sarala, S. 2004, MNRAS, 347, 394

Jarvis, M. J., \& McLure, R. J. 2006, MNRAS, 369, 182

Li, C., Jing, Y. P., Faltenbacher, A., \& Wang, J. 2013, ApJ, 770, L12

Masiero, J., Hodapp, K., Harrington, D., \& Lin, H. 2007, PASP, 119, 1126

Nadathur, S. 2013, MNRAS, 434, 398

Patat, F., \& Romaniello, M. 2006, PASP, 118, 146

Payez, A., Cudell, J. R., \& Hutsemékers, D. 2011, Phys. Rev. D, 84, 085029

Pelgrims, V., \& Cudell, J. R. 2014, MNRAS, 442, 1239

Press, W. H., Teukolsky, S. A., Vetterling, W. T., \& Flannery, B. P. 1992, Numerical Recipes. The Art of Scientific Computing (Cambridge: Cambridge University Press)

Shen, Y., Richards, G. T., \& Strauss, M. A. 2011, ApJS, 194, 45

Shurtleff, R. 2013 [arXiv: 1311.6118]

Simmons, J. F. L., \& Stewart, B. G. 1985, A\&A, 142, 100

Smith, J. E., Robinson, A., Alexander, D. M., et al. 2004, MNRAS, 350, 140

Sluse, D., Hutsemékers, D., Lamy, H., Cabanac, R., \& Quintana, H. 2005, A\&A, 433, 757

Tempel, E., \& Libeskind, N. I. 2013, ApJ, 775, L42

Trowland, H. E., Lewis, G., \& Bland-Hawthorn, J. 2013, ApJ, 762, 72

Wardle, J. F. C., \& Kronberg, P. P. 1974, ApJ, 194, 249

Wills, B. J., \& Browne, I. W. A. 1986, ApJ, 302, 56

Yadav, J. K., Bagla, J. S., \& Khandai, N. 2010, MNRAS, 405, 2009

Zhang, Y., Yang, X., Wang, H., et al. 2013, ApJ, 779, 160 
Table 1. Linear polarization of 93 quasars.

\begin{tabular}{|c|c|c|c|c|c|c|c|c|c|c|c|}
\hline Object & $z$ & LQG & Date & $\begin{array}{r}q \\
(\%)\end{array}$ & $\begin{array}{r}u \\
(\%)\end{array}$ & $\begin{array}{r}p \\
(\%)\end{array}$ & $\begin{array}{r}\sigma_{p} \\
(\%)\end{array}$ & $\begin{array}{r}p_{0} \\
(\%)\end{array}$ & $\begin{array}{c}\theta \\
\left(^{\circ}\right)\end{array}$ & $\begin{array}{l}\sigma_{\theta} \\
\left({ }^{\circ}\right)\end{array}$ & Notes \\
\hline SDSSJ104938.22+214829.3 & 1.2352 & 1 & 2014 Mar. 25 & -0.02 & 0.00 & 0.02 & 0.07 & 0.00 & - & - & \\
\hline SDSSJ105140.40+203921.1 & 1.1742 & 1 & 2014 Mar. 22 & -0.01 & -0.14 & 0.14 & 0.06 & 0.13 & 133 & 13 & \\
\hline SDSSJ105224.08+204634.1 & 1.2032 & 1 & 2014 Mar. 23 & -0.18 & -0.02 & 0.18 & 0.10 & 0.16 & 93 & 18 & \\
\hline SDSSJ105258.16+201705.4 & 1.2526 & 1 & 2014 Mar. 23 & -0.09 & 0.24 & 0.26 & 0.21 & 0.19 & 55 & 32 & \\
\hline SDSSJ105421.90+212131.2 & 1.2573 & 1 & 2014 Mar. 23 & -1.04 & -0.09 & 1.04 & 0.08 & 1.04 & 93 & 2 & \\
\hline SDSSJ105446.73+195710.5 & 1.2195 & 1 & 2014 Mar. 22 & -1.64 & 0.93 & 1.89 & 0.23 & 1.88 & 75 & 4 & \\
\hline SDSSJ105525.18+191756.3 & 1.2005 & 1 & 2014 Mar. 23 & -0.16 & 0.34 & 0.38 & 0.13 & 0.36 & 58 & 10 & \\
\hline SDSSJ105556.22+184718.4 & 1.2767 & 1 & 2014 Mar. 23 & 0.08 & 0.02 & 0.08 & 0.12 & 0.00 & - & - & \\
\hline SDSSJ105611.27+170827.5 & 1.3316 & 1 & 2014 Mar. 25 & 0.01 & 1.29 & 1.29 & 0.08 & 1.29 & 45 & 2 & \\
\hline SDSSJ105714.02+184753.3 & 1.2852 & 1 & 2014 Mar. 24 & -0.02 & -0.05 & 0.05 & 0.09 & 0.00 & - & - & \\
\hline SDSSJ105805.09+200341.0 & 1.2731 & 1 & 2014 Mar. 23 & 0.13 & 0.17 & 0.21 & 0.09 & 0.19 & 26 & 13 & \\
\hline SDSSJ105832.01+170456.0 & 1.2813 & 1 & 2014 Mar. 22 & -0.35 & -0.09 & 0.36 & 0.16 & 0.33 & 97 & 14 & \\
\hline SDSSJ105840.49+175415.5 & 1.2687 & 1 & 2014 Mar. 24 & 0.14 & -0.24 & 0.28 & 0.11 & 0.26 & 150 & 12 & \\
\hline SDSSJ105928.57+164657.9 & 1.2993 & 1 & 2014 Mar. 24 & 0.02 & 0.24 & 0.24 & 0.15 & 0.20 & 43 & 21 & \\
\hline SDSSJ110016.88+193624.7 & 1.2399 & 1 & 2014 Mar. 22 & 0.88 & -0.72 & 1.14 & 0.23 & 1.12 & 160 & 6 & \\
\hline SDSSJ110039.99+165710.3 & 1.2997 & 1 & 2014 Mar. 22 & -0.06 & 0.16 & 0.17 & 0.12 & 0.14 & 56 & 25 & \\
\hline SDSSJ104139.15+143530.2 & 1.2164 & 2 & 2014 Mar. 22 & -0.21 & 0.14 & 0.25 & 0.16 & 0.21 & 73 & 22 & \\
\hline SDSSJ104321.62+143600.2 & 1.2660 & 2 & 2014 Mar. 24 & -0.19 & -0.11 & 0.22 & 0.10 & 0.20 & 105 & 14 & \\
\hline SDSSJ104430.92+160245.0 & 1.2294 & 2 & 2014 Mar. 22 & -0.08 & 0.04 & 0.09 & 0.07 & 0.07 & 76 & 30 & \\
\hline SDSSJ104445.03+151901.6 & 1.2336 & 2 & 2014 Mar. 23 & 1.13 & -0.53 & 1.25 & 0.11 & 1.25 & 168 & 3 & \\
\hline SDSSJ104520.62+141724.2 & 1.2650 & 2 & 2014 Mar. 23 & 0.19 & 0.01 & 0.19 & 0.12 & 0.16 & 1 & 22 & \\
\hline SDSSJ104604.05+140241.2 & 1.2884 & 2 & 2014 Mar. 22 & -0.17 & 0.50 & 0.53 & 0.15 & 0.51 & 54 & 8 & \\
\hline SDSSJ104616.31+164512.6 & 1.2815 & 2 & 2014 Mar. 24 & -1.24 & 0.13 & 1.25 & 0.11 & 1.25 & 87 & 3 & \\
\hline SDSSJ104624.25+143009.1 & 1.3620 & 2 & 2014 Mar. 23 & -0.04 & -0.20 & 0.20 & 0.10 & 0.18 & 129 & 16 & \\
\hline SDSSJ104813.63+162849.1 & 1.2905 & 2 & 2014 Mar. 22 & -0.04 & 0.16 & 0.17 & 0.12 & 0.14 & 53 & 25 & \\
\hline SDSSJ104859.74+125322.3 & 1.3597 & 2 & 2014 Mar. 25 & -0.02 & 0.72 & 0.72 & 0.13 & 0.71 & 46 & 5 & \\
\hline SDSSJ104915.66+165217.4 & 1.3459 & 2 & 2014 Mar. 23 & -0.14 & -0.51 & 0.53 & 0.09 & 0.52 & 127 & 5 & \\
\hline SDSSJ104922.60+154336.1 & 1.2590 & 2 & 2014 Mar. 22 & -0.26 & 0.03 & 0.26 & 0.15 & 0.22 & 87 & 19 & \\
\hline SDSSJ104924.30+154156.0 & 1.2965 & 2 & 2014 Mar. 25 & -0.14 & 0.16 & 0.21 & 0.12 & 0.18 & 66 & 19 & \\
\hline SDSSJ104941.67+151824.6 & 1.3390 & 2 & 2014 Mar. 24 & 0.51 & -1.21 & 1.31 & 0.13 & 1.30 & 146 & 3 & \\
\hline SDSSJ104947.77+162216.6 & 1.2966 & 2 & 2014 Mar. 22 & 0.13 & -0.01 & 0.13 & 0.14 & 0.00 & - & - & \\
\hline SDSSJ104954.70+160042.3 & 1.3373 & 2 & 2014 Mar. 23 & -0.21 & -0.29 & 0.36 & 0.17 & 0.32 & 117 & 15 & \\
\hline SDSSJ105001.22+153354.0 & 1.2500 & 2 & 2014 Mar. 25 & -0.03 & -0.02 & 0.03 & 0.11 & 0.00 & - & - & \\
\hline SDSSJ105042.26+160056.0 & 1.2591 & 2 & 2014 Mar. 25 & 0.01 & 0.20 & 0.20 & 0.08 & 0.18 & 44 & 12 & \\
\hline SDSSJ105104.16+161900.9 & 1.2502 & 2 & 2014 Mar. 22 & 0.24 & -0.01 & 0.24 & 0.10 & 0.22 & 179 & 13 & \\
\hline SDSSJ105117.00+131136.0 & 1.3346 & 2 & 2014 Mar. 23 & -0.37 & 0.46 & 0.59 & 0.22 & 0.55 & 64 & 11 & \\
\hline SDSSJ105119.60+142611.4 & 1.3093 & 2 & 2014 Mar. 25 & 0.18 & 0.03 & 0.18 & 0.10 & 0.16 & 5 & 18 & \\
\hline SDSSJ105122.98+115852.3 & 1.3085 & 2 & 2014 Mar. 23 & 0.01 & -0.02 & 0.02 & 0.10 & 0.00 & - & - & \\
\hline SDSSJ105125.72+124746.3 & 1.2810 & 2 & 2014 Mar. 25 & 0.08 & -0.06 & 0.10 & 0.08 & 0.07 & 160 & 31 & RadioS \\
\hline SDSSJ105132.22+145615.1 & 1.3607 & 2 & 2014 Mar. 22 & -0.40 & 0.15 & 0.43 & 0.10 & 0.42 & 80 & 7 & \\
\hline SDSSJ105144.88+125828.9 & 1.3153 & 2 & 2014 Mar. 25 & 0.21 & 0.13 & 0.25 & 0.07 & 0.24 & 15 & 8 & RadioS \\
\hline SDSSJ105210.02+165543.7 & 1.3369 & 2 & 2014 Mar. 24 & -0.16 & -0.23 & 0.28 & 0.09 & 0.27 & 117 & 10 & \\
\hline SDSSJ105222.13+123054.1 & 1.3162 & 2 & 2014 Mar. 24 & -0.08 & 0.32 & 0.33 & 0.13 & 0.31 & 52 & 12 & \\
\hline SDSSJ105223.68+140525.6 & 1.2483 & 2 & 2014 Mar. 22 & 0.27 & 0.41 & 0.49 & 0.11 & 0.48 & 28 & 7 & \\
\hline SDSSJ105245.80+134057.4 & 1.3544 & 2 & 2014 Mar. 25 & 0.65 & 1.15 & 1.32 & 0.11 & 1.32 & 30 & 2 & RadioS \\
\hline SDSSJ105257.17+105933.5 & 1.2649 & 2 & 2014 Mar. 24 & 0.54 & -0.15 & 0.56 & 0.11 & 0.55 & 172 & 6 & \\
\hline SDSSJ105412.67+145735.2 & 1.2277 & 2 & 2014 Mar. 24 & -0.18 & -0.32 & 0.37 & 0.10 & 0.36 & 121 & 8 & RadioS \\
\hline SDSSJ105435.64+101816.3 & 1.2600 & 2 & 2014 Mar. 24 & -0.03 & 0.09 & 0.10 & 0.08 & 0.07 & 55 & 31 & \\
\hline SDSSJ105442.71+104320.6 & 1.3348 & 2 & 2014 Mar. 24 & 0.71 & -0.18 & 0.73 & 0.11 & 0.72 & 173 & 4 & \\
\hline SDSSJ105523.03+130610.7 & 1.3570 & 2 & 2014 Mar. 25 & 0.03 & 0.00 & 0.03 & 0.10 & 0.00 & - & - & \\
\hline SDSSJ105525.68+113703.0 & 1.2893 & 2 & 2014 Mar. 24 & -0.36 & 2.52 & 2.55 & 0.10 & 2.55 & 49 & 1 & RadioS \\
\hline SDSSJ105541.83+111754.2 & 1.3298 & 2 & 2014 Mar. 25 & -0.45 & 0.01 & 0.45 & 0.11 & 0.44 & 89 & 7 & \\
\hline SDSSJ105621.90+143401.0 & 1.2333 & 2 & 2014 Mar. 23 & -0.42 & 0.13 & 0.44 & 0.13 & 0.42 & 82 & 9 & \\
\hline SDSSJ105637.49+150047.5 & 1.3713 & 2 & 2014 Mar. 25 & -0.37 & 0.46 & 0.59 & 0.16 & 0.57 & 65 & 8 & \\
\hline SDSSJ105637.98+100307.2 & 1.2730 & 2 & 2014 Mar. 23 & -0.08 & 0.16 & 0.18 & 0.12 & 0.15 & 58 & 23 & \\
\hline SDSSJ105655.36+144946.2 & 1.2283 & 2 & 2014 Mar. 24 & -0.14 & -0.05 & 0.15 & 0.13 & 0.10 & 99 & 39 & \\
\hline
\end{tabular}

Notes. Column 1 gives the quasar SDSS name, Col. 2 the redshift $z$, Col. 3 the quasar group (1, 2, 3 = Huge-LQG, 4 = CCLQG; see also Fig. 4), Col. 4 the observation date, Cols. 5, 6 the $q$ and $u$ normalized Stokes parameters corrected for the offset angle, Col. 7 the polarization degree $p$, Col. 8 the error on the polarization degree $\sigma_{p} \simeq \sigma_{q} \simeq \sigma_{u}$, Col. 9 the polarization degree $p_{0}$ debiased according to the Wardle \& Kronberg (1974) method (see also Simmons \& Stewart 1985), Col. 10 the polarization position angle $\theta$, defined between $0^{\circ}$ and $180^{\circ}$ and measured in the equatorial coordinate system (north $=0^{\circ}$ and east $=90^{\circ}$ ), and Col. 11 the error on the polarization angle estimated using $\sigma_{\theta}=28.65^{\circ} \sigma_{p} / p_{0}$ to avoid underestimation at low signal-to-noise (Wardle \& Kronberg 1974). Additional characteristics of the targets were retrieved from the NASA/IPAC Extragalactic Database (NED) and given in Col. 12: radio-source (RadioS), broad absorption line (BAL) quasar, and low-ionization BAL (LoBAL) quasar. 
D. Hutsemékers et al.: Alignment of quasar polarizations with large-scale structures

Table 1. continued.

\begin{tabular}{|c|c|c|c|c|c|c|c|c|c|c|c|}
\hline Object & $z$ & LQG & Date & $\begin{array}{r}q \\
(\%)\end{array}$ & $\begin{array}{r}u \\
(\%)\end{array}$ & $\begin{array}{r}p \\
(\%)\end{array}$ & $\begin{array}{c}\sigma_{p} \\
(\%)\end{array}$ & $\begin{array}{r}p_{0} \\
(\%)\end{array}$ & $\begin{array}{c}\theta \\
\left(^{\circ}\right)\end{array}$ & $\begin{array}{l}\sigma_{\theta} \\
\left(^{\circ}\right)\end{array}$ & Notes \\
\hline SDSSJ105855.33+081350.7 & 1.2450 & 3 & 2014 Mar. 23 & -0.01 & 0.29 & 0.29 & 0.09 & 0.28 & 46 & 9 & \\
\hline SDSSJ110006.02+092638.7 & 1.2485 & 3 & 2014 Mar. 22 & 0.17 & 0.14 & 0.22 & 0.09 & 0.20 & 19 & 13 & \\
\hline SDSSJ110148.66+082207.1 & 1.1940 & 3 & 2014 Mar. 24 & 0.10 & -0.19 & 0.22 & 0.14 & 0.18 & 149 & 22 & \\
\hline SDSSJ110217.19+083921.1 & 1.2355 & 3 & 2014 Mar. 23 & 0.35 & -0.02 & 0.35 & 0.12 & 0.33 & 178 & 10 & \\
\hline SDSSJ110504.46+084535.3 & 1.2371 & 3 & 2014 Mar. 25 & 0.10 & -0.10 & 0.14 & 0.10 & 0.11 & 157 & 26 & \\
\hline SDSSJ110621.40+084111.2 & 1.2346 & 3 & 2014 Mar. 22 & 0.09 & 0.10 & 0.14 & 0.15 & 0.00 & - & - & \\
\hline SDSSJ110736.60+090114.7 & 1.2266 & 3 & 2014 Mar. 22 & -0.42 & 0.08 & 0.43 & 0.11 & 0.42 & 85 & 8 & RadioS \\
\hline SDSSJ110744.61+095526.9 & 1.2228 & 3 & 2014 Mar. 25 & 0.44 & 0.25 & 0.51 & 0.08 & 0.50 & 15 & 5 & \\
\hline SDSSJ111007.89+104810.3 & 1.2097 & 3 & 2014 Mar. 23 & 0.01 & 0.55 & 0.55 & 0.12 & 0.54 & 45 & 6 & \\
\hline SDSSJ111009.58+075206.8 & 1.2123 & 3 & 2014 Mar. 24 & 0.67 & 1.68 & 1.81 & 0.17 & 1.80 & 34 & 3 & \\
\hline SDSSJ111416.17+102327.5 & 1.2053 & 3 & 2014 Mar. 26 & 0.26 & 0.02 & 0.26 & 0.08 & 0.25 & 3 & 9 & \\
\hline SDSSJ111545.30+081459.8 & 1.1927 & 3 & 2014 Mar. 24 & -0.30 & 0.16 & 0.34 & 0.14 & 0.31 & 76 & 13 & \\
\hline SDSSJ111802.11+103302.4 & 1.2151 & 3 & 2014 Mar. 23 & 1.01 & -3.84 & 3.97 & 0.10 & 3.97 & 142 & 1 & \\
\hline SDSSJ111823.21+090504.9 & 1.1923 & 3 & 2014 Mar. 24 & -0.10 & -0.01 & 0.10 & 0.18 & 0.00 & - & - & \\
\hline SDSSJ112019.62+085905.1 & 1.2239 & 3 & 2014 Mar. 23 & 0.38 & -0.15 & 0.41 & 0.13 & 0.39 & 170 & 10 & \\
\hline SDSSJ112059.27+101109.2 & 1.2103 & 3 & 2014 Mar. 24 & 0.44 & 0.29 & 0.53 & 0.22 & 0.49 & 17 & 13 & \\
\hline SDSSJ112109.76+075958.6 & 1.2369 & 3 & 2014 Mar. 25 & -0.01 & 0.33 & 0.33 & 0.08 & 0.32 & 46 & 7 & \\
\hline SDSSJ104114.06+034312.0 & 1.2633 & 4 & 2014 Mar. 26 & -0.26 & 0.41 & 0.49 & 0.16 & 0.46 & 61 & 10 & BAL \\
\hline SDSSJ104115.58+051345.0 & 1.2553 & 4 & 2014 Mar. 26 & -0.14 & 0.27 & 0.30 & 0.13 & 0.27 & 59 & 14 & \\
\hline SDSSJ104116.79+035511.4 & 1.2444 & 4 & 2014 Mar. 26 & -1.46 & -0.51 & 1.55 & 0.11 & 1.55 & 100 & 2 & LoBAL \\
\hline SDSSJ104225.63+035539.1 & 1.2293 & 4 & 2014 Mar. 26 & 0.48 & 0.50 & 0.69 & 0.08 & 0.69 & 23 & 3 & \\
\hline SDSSJ104256.38+054937.4 & 1.3555 & 4 & 2014 Mar. 26 & 0.20 & -0.36 & 0.41 & 0.12 & 0.39 & 149 & 9 & \\
\hline SDSSJ104425.80+060925.6 & 1.2523 & 4 & 2014 Mar. 26 & -0.36 & -0.20 & 0.41 & 0.11 & 0.40 & 105 & 8 & BAL \\
\hline SDSSJ104637.30+075318.7 & 1.3635 & 4 & 2014 Mar. 26 & 0.04 & 0.09 & 0.10 & 0.07 & 0.08 & 34 & 25 & RadioS \\
\hline SDSSJ104733.16+052454.9 & 1.3341 & 4 & 2014 Mar. 26 & 0.04 & -0.19 & 0.19 & 0.07 & 0.18 & 142 & 11 & \\
\hline SDSSJ105010.05+043249.1 & 1.2158 & 4 & 2014 Mar. 26 & -2.46 & -1.04 & 2.67 & 0.08 & 2.67 & 102 & 1 & RadioS \\
\hline SDSSJ105018.10+052826.4 & 1.3067 & 4 & 2014 Mar. 26 & -0.11 & 0.24 & 0.26 & 0.10 & 0.24 & 57 & 12 & \\
\hline SDSSJ105422.47+033719.3 & 1.2278 & 4 & 2014 Mar. 26 & -0.18 & -0.28 & 0.33 & 0.11 & 0.31 & 118 & 10 & RadioS \\
\hline SDSSJ105423.26+051909.8 & 1.2785 & 4 & 2014 Mar. 26 & 0.13 & 0.22 & 0.26 & 0.09 & 0.25 & 30 & 11 & BAL \\
\hline SDSSJ105512.23+061243.9 & 1.3018 & 4 & 2014 Mar. 26 & -0.61 & -0.77 & 0.98 & 0.12 & 0.97 & 116 & 4 & \\
\hline SDSSJ105534.66+033028.8 & 1.2495 & 4 & 2014 Mar. 26 & -0.09 & 0.38 & 0.39 & 0.09 & 0.38 & 52 & 7 & \\
\hline SDSSJ105537.63+040520.0 & 1.2619 & 4 & 2014 Mar. 26 & -0.10 & 0.02 & 0.10 & 0.11 & 0.00 & - & - & \\
\hline SDSSJ105719.23+045548.2 & 1.3355 & 4 & 2014 Mar. 26 & -0.36 & 0.44 & 0.57 & 0.13 & 0.56 & 65 & 7 & RadioS \\
\hline SDSSJ105821.28+053448.9 & 1.2540 & 4 & 2014 Mar. 26 & 0.09 & 0.06 & 0.11 & 0.10 & 0.06 & 15 & 47 & \\
\hline SDSSJ105833.86+055440.2 & 1.3222 & 4 & 2014 Mar. 25 & 0.15 & 0.60 & 0.62 & 0.21 & 0.59 & 38 & 10 & \\
\hline SDSSJ110108.00+043849.6 & 1.2516 & 4 & 2014 Mar. 25 & 0.52 & 0.66 & 0.84 & 0.10 & 0.83 & 26 & 3 & \\
\hline SDSSJ110412.00+044058.2 & 1.2554 & 4 & 2014 Mar. 25 & 0.15 & -0.08 & 0.17 & 0.11 & 0.14 & 167 & 22 & \\
\hline
\end{tabular}

